„Sensibilisierend“ wirken solche fremden Bausteine, in denen der optische Absorptionsvorgang so verläuft, daß zwar bewegliche Elektronen geliefert, aber keinen zum ungestörten Gitterverband gehörenden Anionen Elektronen weggenommen werden, daß also keine Defektelektronen (Elektronenfehlstellen oder holes) im Gitter entstehen. Als gutes Beispiel derartiger Sensibilisatoren nennen wir für Kaliumchlorid kleine Beimengungen von $\mathrm{K}_{2} \mathrm{O}$, für Silberbromid kleine Beimengungen von $\mathrm{Ag}_{2} \mathrm{~S}$. Beide Sensibilisatoren sind im Kristallgitter durch ihr optisches Absorptionsspektrum nachzuweisen. Doch sind auch hier die Verhältnisse in der Modellsubstanz $\mathrm{KCl}$ mit $\mathrm{K}_{2} \mathrm{O}$ günstiger ${ }^{9}$ als beim $\mathrm{AgBr}$ mit $\mathrm{Ag}_{2} \mathrm{~S}^{10}$. Das zeigt die Abb. 5, in der zwar im $\mathrm{KCl}$, aber nicht im $\mathrm{AgBr}$, die Absorption des $\mathrm{Zu}$ satzes gut von der Grundgitterabsorption getrennt ist.

Beim $\mathrm{AgBr}$ mit $\mathrm{Ag}_{2} \mathrm{~S}$ deutet man den Verlauf des photochemischen Primärprozesses etwa folgender-

9 R. H a h n, Diplomarbeit Göttingen, unveröffentlicht. S. A k p i n a r, Ann. Physik (5) 37, 429 [1940].

10 O. S t a s i w u. J. T e l t o w, Nachr. Ges. Wiss. Göttingen, math.-physik. Kl. 1941, 100.

11 O. S t a s i w, Z. Physik 130, 39 [1951]. maßen: Die vom Sensibilisator absorbierten Lichtquanten liefern bewegliche Elektronen. Es entstehen einwertige Schwefelionen $\left(\mathrm{S}^{-}\right)$. Diese spielen nunmehr die Rolle normaler Gitteranionen, es entstehen also keine Defektelektronen. Die Elektronen werden zunächst in einer Bindung festgelegt, die der der F-Zentren in den Alkalihalogeniden zum mindesten ähnlich ist. Diese Bindung hat nur bei kleinen Temperaturen große Lebensdauer. Dann kann man auch das zugehörige Absorptionsspektrum beobachten. Man findet es in Abb. 6 ( $\mathrm{S}$ t a s i w ${ }^{11}$ ). Bei großen Temperaturen vereinigen sich die „F-Zentren“ zu Assoziaten (Kolloiden). Man erhält die schon lange bekannte Bande, die in Abb. 4 reproduziert ist.

Zusammenfassend und rückblickend kann man also sagen: Die Eignung des $\mathrm{AgBr}$ als photographische Grundsubstanz verdankt man vor allem der Tatsache, daß die photochemischen Primärprodukte sich leicht zu Assoziaten vereinigen. Die Wirksamkeit des Sensibilisators $\left(\mathrm{Ag}_{2} \mathrm{~S}\right)$ beruht darauf, daß bei der optischen Dissoziation nur eine Sorte beweglicher Partner gebildet wird.

\title{
Zwei neue experimentelle Belege für die gemischte Fehlordnung und die Störstellenassoziation in Silberbromid
}

\author{
Von O. Stasiw und J. Teltow \\ Aus dem Institut für Festkörperforschung der Deutschen Akademie der Wissenschaften zu Berlin, \\ Zweigstelle Dresden \\ (Z. Naturforschg. 6 a 363-366 [1951]; eingegangen am 24. April 1951) \\ John Eggert zum 60. Geburtstag gewidmet
}

\begin{abstract}
Die Schwärzung von AgBr-Gelatine-Emulsionen, die bei $-190^{\circ} \mathrm{C}$ belichtet wurden, hängt von der Lagerungszeit zwischen Belichtung und Entwicklung und von der Art des Entwicklers ab. AgBr-Kristalle mit $\mathrm{Ag}_{2} \mathrm{~S}-\mathrm{Zusatz}$, die bei $-190^{\circ}$ fluoreszieren, zeigen bei bestimmter Wärmevorbehandlung ein Nachleuchten. Beide Effekte werden mit Hilfe des Fehlordnungsmodells gedeutet.
\end{abstract}

So unzweifelhaft die Gesamtbilanz der photochemischen Zersetzung von Silberbromid seit dem Nachweis der Gültigkeit des Äquivalenzgesetzes durch Eg g e rt und Noddack ${ }^{1}$ feststand, soviel Mühe und Scharfsinn war in der Folgezeit aufzuwenden, um die einzelnen Phasen dieses Reaktionsmechanismus in ihrer Abhängigkeit von den jeweiligen Bedingungen klar herauszuarbeiten. Entscheidende Fortschritte [1925] in der Klärung dieser Fragen verdankt man einerseits den experimentellen Arbeiten der P o hlschen Schule über Alkalihalogenidkristalle, andererseits den neuen Erkenntnissen vom Realbau der Kristalle, insbesondere der durch Frenkel, Schottky und Wagner entwickelten Vorstellung der Fehlordnung, d. h. einer den thermodynamisch-statistischen Gesetzen unterworfenen Fehlbesetzung einzelner Gitterplätze. Denn gerade diese gittereigenen Fehlstellen spielen im Verein mit gitterfremden Bausteinen die maßgebliche 
Rolle sowohl bei der Ablösung der Photoelektronen wie auch bei ihrer „Kondensation“ mit Silberionen zu einem entwickelungsfähigen Keim im Korn der Emulsion bzw. zu einem etwa $10^{7}$ Atome umfassenden metallischen Ag-Kolloidteilchen beim Print-out-Effekt der Emulsionen oder bei der Verfärbung makroskopischer Kristalle.

Die vorliegende Arbeit will an zwei weiteren Beispielen zeigen, wie es mit Hilfe der Fehlordnungstheorie gelingt, Ordnung in das experimentelle Material $\mathrm{zu}$ bringen und es unter einem einheitlichen Gesichtspunkt zu interpretieren. Deshalb gibt $\S 1$ einen ganz knappen Überblick über die nach dem heutigen Stand unserer Kenntnis in Silberbromid vorhandenen Störstellen und ihre Eigenschaften. In $\$ \S 2$ und 3 werden dann neue Tieftemperaturbeobachtungen zur photographischen Empfindlichkeit von AgBrEmulsionen und zur Lumineszenz von Silberbromidkristallen mit $\mathrm{As}_{2} \mathrm{~S}-\mathrm{Zusatz}$ mitgeteilt und mit Hilfe des Fehlordnungsmodells gedeutet.

\section{§ : Zur Fehlordnung von AgBr}

Zunächst seien die einzelnen Störstellenarten mit einer kurzen Begründung und ihren wichtigsten Eigenschaften aufgezählt. Die Störstellensymbole entsprechen im wesenlichen den Vorschlägen von S chottky. Insbesondere ist mit 'bzw. ' die Überschußladung der betreffenden Störstelle relativ zum Idealgitter bezeichnet. Es bedeuten: $\bigcirc$ Ion auf Zwischengitterplatz, $\square$ durch Entfernung des betreffenden Ions von seinem Gitterplatz gebildete Leerstelle, G Fremdion auf Gitterplatz, das also ein $\mathrm{Ag}^{+}$- bzw. $\mathrm{Br}^{-}$-Ion substituiert hat. Nebeneinandergesetzte Störstellensymbole bezeichnen räumlich unmittelbar benachbarte Störstellenaggregate.

Ag. Silberion auf Zwischengitterplatz, und

$\mathrm{Ag}^{\prime}$ 。 Silberion-Leerstelle. Beide zusammen bedeuten Frenkelsche Fehlordnung im Kationenteilgitter, die als Anteil einer allgemeineren Gesamtfehlordnung experimentell als gesichert betrachtet werden $\mathrm{kann}^{2}$. Konzentration im zusatzfreien $\mathrm{AgBr}$ bei $20^{\circ} \mathrm{C}$ etwa $10^{-6}$, relativ große Beweglichkeit (Diffusionskoeffizient bei $20^{\circ}$ für $\mathrm{Ag}^{\circ}$ 。 etwa $10^{-5}$, für $\mathrm{Ag}^{\prime}$ 。 $10^{-6} \mathrm{~cm}^{2} \mathrm{sec}^{-1}$ ).

$\mathrm{Br}^{\circ}$ 。 Bromion-Leerstelle. Bedeutet mit $\mathrm{Ag}^{\prime}$ 。 zusammen Schottkysche Fehlordnung. Beweglichkeit um etwa 4 Zehnerpotenzen kleiner als bei $\mathrm{Ag}^{\prime}$,

2 J. Te l t ow, Ann. Physik (6) 5, 71 [1949].

3 O. S t a s i w, Z. Physik 127, 522 [1950]. daher durch Abschrecken Einfrieren höherer Gleichgewichtskonzentrationen möglich. Schon bei Raumtemperatur kann die Einstellung von Gleichgewichten, an denen $\mathrm{Br}^{*}$ o beteiligt ist, infolge Diffusionshemmung Stunden in Anspruch nehmen. Die experimentelle Beobachtung solcher Relaxationseffekte, die im Rahmen der Frenkelschen Fehlordnung nicht gedeutet werden können, bildet das Hauptargument zugunsten einer gemischten Fehlordnung in $\mathrm{AgBr}^{3}$.

$\mathrm{S}_{\mathrm{G}}^{\prime} \quad$ Schwefelion auf (Bromion-) Gitterplatz. Nächstliegende Annahme für den Einbau des $\mathrm{S}^{--}$in $\mathrm{AgBr}-\mathrm{Ag}_{2} \mathrm{~S}-\mathrm{Mischkristallen}$ sowie in mit S-haltiger Gelatine gereiften Emulsionen.

$\mathrm{Ag}^{*} \mathrm{~S}_{\mathrm{G}}^{\prime}$ und $\mathrm{S}_{\mathrm{G}}^{\prime} \mathrm{Br}^{\circ}$ 。 assoziierte Störstellen („Komplexe“), zu deren Gunsten (schon infolge elektrostatischer Anziehung) das Gleichgewicht bei $20^{\circ} \mathrm{C}$ und darunter auf Kosten der freien $\mathrm{S}_{\mathrm{G}}^{\prime}$ verschoben ist $^{3}$. Eine rasche Einstellung dieses Gleichgewichts ist aber nur im Falle der $\mathrm{Ag}^{*} \mathrm{~S}_{\mathrm{G}}^{\prime}$ möglich. Der durch $\mathrm{Ag}_{2} \mathrm{~S}-\mathrm{Zusatz}$ in $\mathrm{AgBr}$ bewirkte langwellige Ausläufer der Eigenabsorption ${ }^{4}$ wird - wenigstens bei $20^{\circ} \mathrm{C}$ und darunter - fast ausschließlich den Komplexen $\mathrm{Ag}^{*} \mathrm{~S}_{\mathrm{G}}^{\prime}$ zugeschrieben, da bei diesen Temperaturen freie $S^{\prime}{ }_{G}$ praktisch nicht mehr vorhanden sind und da die Absorption der $\mathrm{S}_{\mathrm{G}} \mathrm{Br}^{\circ}$ 。 vermutlich im Eigenabsorptionsgebiet des AgBr-Gitters liegt ${ }^{5}$.

Anschließend formulieren wir plausible Reaktionsgleichungen für den photochemischen Elementarprozeß im Gebiet der Ausläuferabsorption, bei der also , ein Elektron vom Komplex emittiert wird:

$$
\begin{aligned}
& \mathrm{Ag}_{\circ} \mathrm{S}_{\mathrm{G}}^{\prime}+h v \rightarrow \mathrm{Ag}_{\circ} \mathrm{S}_{\mathrm{G}}+\mathrm{e}^{\prime} \\
& \mathrm{Ag}^{\circ} \circ \mathrm{S}_{\mathrm{G}} \rightarrow \mathrm{Ag}^{\circ} \circ+\mathrm{S}_{\mathrm{G}} \\
& \mathrm{S}_{\mathrm{G}}^{\prime} \mathrm{Br}^{\circ}{ }_{\mathrm{o}}+\mathrm{e}^{\prime} \rightarrow \mathrm{S}_{\mathrm{G}}^{\prime} \mathrm{Br}_{\mathrm{o}} \\
& \mathrm{S}_{\mathrm{G}}^{\prime} \mathrm{Br}_{\mathrm{a}}+\mathrm{Ag}_{\circ}{ }_{\circ} \rightarrow \mathrm{Ag}_{\circ} \mathrm{S}_{\mathrm{G}^{\prime}} \mathrm{Br}_{\mathrm{a}} \text {. }
\end{aligned}
$$

Gemäß der 2. Gleichung bleibt vom Komplex infolge Wegdiffusion des $\mathrm{Ag}^{*}$ 。 nur ein S-Ion mit Elektronendefekt übrig, so daß (infolge Fortfalls der Anziehungskraft des $\mathrm{Ag}^{\circ}{ }_{0}$ ) die Rekombinationswahrscheinlichkeit mit dem Photoelektron stark herabgesetzt ist. Die Gl. (3) beschreibt den Einfang des Photoelektrons. Das Endprodukt in Gl. (4) ist äquivalent einem an $\mathrm{S}_{\mathrm{G}}^{\prime}$ gebundenen neutralen Silberatom,

4 O. St a s iw u. J. T e lt ow, Ann. Physik (5) 40, 181 [1941].

5 O. S t a s i w, Z. Physik 1951, im Druck. 
kann daher als Vorstufe des latenten Bildes aufgefaßt werden. Unterhalb $-120^{\circ} \mathrm{C}$ versagen Gl. (2) und (4) infolge zu geringer Beweglichkeit des $\mathrm{Ag}^{\circ}$. Rekombinationen sind dann häufig, d.h. die Photochemie tritt zugunsten der Lumineszenz in den Hintergrund, wie experimentell beobachtet.

Ein Absorptionsakt im Grundgitter (Photochemie bzw. Fluoreszenz im Eigenabsorptionsgebiet) kann in seinen Auswirkungen auf die Gl. (2)-(4) zurückgeführt werden, wenn man annimmt, daß die Anregungsenergie strahlungslos auf den $\mathrm{Ag}^{*}{ }^{\circ} \mathrm{S}_{\mathrm{G}}$-Komplex übertragen wird oder daß dieser das Defektelektron bindet. Doch ist hierbei eine schlechtere Ausbeute zu erwarten, da Photo- und Defektelektron vorher rekombinieren können.

Eine derartige Vorstufe des latenten Bildes ist bei den Alkalihalogeniden schon seit längerer Zeit bekannt ${ }^{6}$ : es sind die atomaren Farbzentren, die durch geeignete Wärmebehandlung zu groben, „kolloidalen“ Metallpartikeln zusammenflocken. Bei den Silberhalogeniden jedoch wurde ein solches atomares Vorstadium erst kürzlich ${ }^{5}$ experimentell greifbar: Bei Belichtung von $\mathrm{AgBr}-\mathrm{Kristallen}$ mit $\mathrm{Ag}_{2} \mathrm{~S}-\mathrm{Zusatz}$ bei $-120^{\circ}$ entstehen neue, bisher nicht beobachtete Absorptionsbanden im sichtbaren Spektralgebiet. Sie lassen sich den in (3) und (4) auftretenden Komplexen zuordnen; der: Mechanismus der Bildung der einzelnen Banden und ihre Temperaturabhängigkeit sind dann mit den Gln. (3) und (4) vollständig im Einklang.

\section{\$2: Zur photographischen Empfindlich- keit von AgBr-Emulsionen bei $-190^{\circ}$}

Handelsübliches Bromsilberpapier (in unserem Falle Mimosa Luxus Bromosa L 301) wird mit der Hg-Linie $4.36 \mathrm{~m} \mu$ (Hg-Niederdrucklampe, spektrale Zerlegung) bei gleichbleibender Intensität und Belichtungszeit (etwa $1 / 10 \mathrm{sec}$ ) bestrahlt und unter einheitlichen Bedingungen entwickelt.

Die Abhängigkeit der Schwärzung von a) der Temperatur, bei der die Belichtung erfolgte, b) der Lagerungszeit zwischen Belichtung und Entwicklung, c) normaler bzw. Tiefenentwicklung (T.E.) mittels Chromsäurebads und Oxalatentwicklers ${ }^{7}$, ist in Tab. 1 wiedergegeben. Quantitave Angaben folgen in späteren Arbeiten, in denen u. a. der Intensitätseinfluß untersucht werden soll.

6 R. W. P o h l, Physik. Z. 39, 36 [1938] (zusammenfassender Bericht).

7 R. D e b ot, Bull. Soc. Sci. Liège 10, Nr. 1, 90 [1941]. Vgl. den Bericht von I. M. Kelle r u. K. Ma etzig, Z. wiss. Photogr. 43, 138 [1948].

\begin{tabular}{|c|c|c|c|}
\hline Nr. & $\begin{array}{l}\text { Tempe- } \\
\text { ratur } \\
\text { bei der } \\
\text { Belichtg. }\end{array}$ & $\begin{array}{l}\text { Entwicklung } \\
\text { erfolgt }\end{array}$ & Schwärzung \\
\hline 1 & $20^{\circ}$ & Sofort & Normal \\
\hline 2 & $20^{\circ}$ & $\begin{array}{l}\text { Nach einigen } \\
\text { Tagen }\end{array}$ & $\begin{array}{l}\text { Normal, kein Unter- } \\
\text { schied gegen } 1\end{array}$ \\
\hline 3 & $-190^{\circ}$ & Sofort & $\begin{array}{l}\text { Etwa 5-mal kleiner als } \\
\text { bei 1, d.h. gleiche } \\
\text { Schwärzung erfordert } \\
\text { 5-fache Belichtungszeit }\end{array}$ \\
\hline 4 & $-190^{\circ}$ & $\begin{array}{l}\text { Nach einigen } \\
\text { Tagen }\end{array}$ & $\begin{array}{l}\text { Steigt mit der Lage- } \\
\text { rungszeit an, erreicht } \\
\text { nach } 7 \text { Tagen die Hälfte } \\
\text { des Normalwertes von } 1\end{array}$ \\
\hline 5 & $20^{\circ}$. & Sofort, T.E. & $\begin{array}{l}\text { Sehr gering, etwa } 1 / 20 \\
\text { der normalen Schwär- } \\
\text { zung von } 1\end{array}$ \\
\hline 6 & $-190^{\circ}$ & Sofort, T.E. & $\begin{array}{l}\text { Keine Änderung gegen- } \\
\text { über } 3\end{array}$ \\
\hline 7 & $-190^{\circ}$ & $\begin{array}{l}\text { Nach einigen } \\
\text { Tagen, T.E. }\end{array}$ & Sehr gering, wie 5 \\
\hline
\end{tabular}

Tab. 1. Abhängigkeit der Schwärzung von Temperatur, Lagerungszeit und Entwicklung.

D e utung: Es werde angenommen, daß auch im Kristallgitter des Emulsionskorns komplexe Störstellen mit ähnlichen Eigenschaften vorliegen, wie wir sie im $\mathrm{AgBr}-\mathrm{Kristall}$ mit $\mathrm{Ag}_{2} \mathrm{~S}-\mathrm{Zusatz}$ annehmen, mit Schwefel oder vielleicht auch Sauerstoff als „Komplexbildner"; sie dürften bei der Reifung entstehen.

Infolge der Verschiebung nach kürzeren Wellen, die sowohl Eigenabsorption wie Ausläufer mit abnehmender Temperatur erfahren, wird das 436-m $\mu$-Licht bei Zimmertemperatur hauptsächlich im Grundgitter, bei $-190^{\circ}$ dagegen zu einem erheblichen Anteil von diesen Störstellen absorbiert.

Bei $20^{\circ}$ entstehen demnach primär neben den Photoelektronen Defektelektronen in fast gleicher Anzahl, die die Ausbeute verringern, ausgenommen das Gebiet nahe der Kornoberfläche, wo die Gelatine sie einfangen kann. Nur in diesem Gebiet können daher die Photoelektronen unbehindert nach Gln. (3) und (4) reagieren, daher entsteht bei $20^{\circ}$ das latente Bild vorwiegend an der Kornoberfläche (Versuch 5).

Bei $-190^{\circ}$ dagegen dürfte die „BromakzeptorWirkung" der Gelatine versagen. Daher ist das bei $-190^{\circ}$ entstandene latente Bild zwar schwächer (Versuch 3), aber homogen im Korn verteilt (Versuch 6); es rührt im wesentlichen nur noch von den Absorptionsakten an den komplexen Störstellen her. Bei Lagerung können die entsprechend Gl. (4) gebildeten Vorkeime an die Oberfläche diffundieren und so eine 
Empfindlichkeitssteigerung bewirken (Versuch 4) ${ }^{8}$, die sich allerdings nur bei normaler Oberflächenentwicklung und nicht bei Tiefenentwicklung bemerkbar macht (Versuch 7).

\section{§3: Lumineszenz von AgBr-Kristallen mit $\mathrm{Ag}_{2} \mathrm{~S}-\mathrm{Zus}$ atz}

Aus einer im Gang befindlichen größeren Untersuchung über die Abhängigkeit der AgBr-Lumineszenz von Zusätzen seien nur die folgenden experimentellen Befunde herausgegriffen:

Der hohen photochemischen Empfindlichkeit, die etwa 0,02 Mol.-\% $\mathrm{Ag}_{2} \mathrm{~S}$ in AgBr-Kristallen hervorrufen, entspricht eine intensive gelbe Lumineszenz bei $-190^{\circ}$. Diese Lumineszenz hängt jedoch entscheidend von der Wärmevorbehandlung ab. Werden nämlich die Kristalle nach Temperung bei $+380^{\circ}$ von dieser Temperatur unmittelbar auf $-190^{\circ}$ abgeschreckt, so fluoreszieren sie intensiv ohne eine Spur von Nach-

8 Zur erleichterten Diffusion assoziierter Störstellen vgl. auch: E. S c hön e, O. S t a s i w u. J. T el tow, Z. physik. Chem. 197, 145 [1951].

9 I. T e l t ow, Z. physik. Chem., Abt. (B) 195, 213 [1950]. leuchten. Schreckt man dagegen in 2 Stufen ab, mit einer eingeschalteten Lagerungszeit bei Zimmertemperatur, so zeigt sich neben der gelben Fluoreszenz ein deutliches Nachleuchten, das sowohl durch Rotlicht wie durch Erwärmung ausgeleuchtet werden kann. Die Helligkeit des Nachleuchtens nimmt mit der Lagerungsdauer von einigen Minuten bis zu einigen Stunden $\mathrm{zu}$, danach infolge Entmischungserscheinungen ${ }^{9}$ wieder $a b$.

D e u tung: Aus den in $\S 1$ erwähnten Beweglichkeiten der Störstellen geht hervor, daß die $\mathrm{S}_{\mathrm{G}}^{\prime} \mathrm{Br}^{\circ}{ }_{{ }^{-}}$Komplexe zu ihrer Bildung bei Zimmertemperatur eine Zeitdauer von Minuten bis Stunden benötigen. Sie sind daher in den gelagerten Kristallen vorhanden, fehlen jedoch in den von $380^{\circ}$ auf $-190^{\circ}$ abgeschreckten. Nimmt man an, daß diese Komplexe entsprechend Gl. (3) als Speicher (Haftstellen) für die Photoelektronen wirken, so ist damit erklärt, warum nur gelagerte Kristalle phosphoreszieren. Zugleich ergibt sich die Folgerung, daß die auch in den ungelagerten Kristallen noch vorhandenen Störstellen, insbesondere $\mathrm{Br}^{*}$ o und $\mathrm{Ag}^{*}{ }_{\circ} \mathrm{S}_{\mathrm{G}}$, nicht Photoelektronen einzufangen und zu binden vermögen.

\title{
Spectral Sensitivity and Chemical Sensitization of Photographic Emulsions
}

\author{
By Martin Biltz \\ Communication No. 1395 from the Kodak Research Laboratories, Rochester, New York, USA. \\ (Z. Naturforschg. 6 a, 366-368 [1951]; eingegangen am 16. April 1951) \\ To Professor John Eggert for his $60^{\text {th }}$ birthday
}

The article describes some earlier results and a few recent ones of experiments on the spectral sensitivity of photographic emulsions and considers the consequences of these results, especially with regard to the action of chemical sensitizers.

$\mathrm{O}$ n April 4, 1938, J. Eg gert gave a lecture before the Faraday Society in Bristol on "The Spectral Sensitivity of Photographic Layers"1. This lecture was based on experiments carried out in the „Wissenschaftliches Zentral-Laboratorium der Photographischen Abteilung der I.G. Farbenindustrie A.-G. (Agfa)" of which he was director at that time. The first part of that paper is entitled „The Spectral Sensitivity of Unsensitized Layers", a heading which

$1 \mathrm{~J}$. Eggert and M. Biltz, Trans. Faraday Soc. 34, 892 [1938]; Veröff. wiss. Zentr.-Lab. photogr. Abt. Agfa 6, 23 [1939]; Z. wiss. Photogr. Photophysik Photochem. 39, 140 [1940]. might be considered to be rather strange, even contradictory. How can something which is not sensitized be sensitive? However, apparently everyone in the audience was well versed in photographic science, and therefore, no one had any doubt as to the subject which Professor Eggert was treating. He wished to exclude photographic emulsions containing organic dyes which absorb light outside the absorption region of the silver halides, and extend the spectral sensitivity of the emulsions to longer wave lengths. $\mathrm{He}$ did not exclude chemically sensitized emulsions, i.e., emulsions the sensitivity of which is increased by substances which do not appreciably increase their 